\title{
e-Voting System: Technologies and Implementations
}

\author{
Madona Raichel \\ Computer Science and Engineering \\ Mar Baselios College of Engineering and \\ Technology \\ Trivandrum, India
}

\author{
Anitha Sandeep \\ Computer Science and Engineering \\ Mar Baselios College of Engineering and \\ Technology \\ Trivandrum, India
}

\begin{abstract}
The exponential headway in data and electronic correspondence has offered climb to new applications that were inconceivable only few years prior. One of the primary applications is in the region of voting. There exists an opportunity and inspiration to execute such an application with the reason for offering voters a plausibility of e voting at private or government race particularly for NRIs. The real playing point is significant diminishment in the expense and money of race process and significant impediment is stage similarity.
\end{abstract}

\section{General Terms}

Anonymous communication, Security constraints, e-voting module

\section{Keywords}

e-voting, privacy, anonymous identity, multiparty computation

\section{INTRODUCTION}

The utilization of web as a communication medium for business or individual necessities depends to some degree on its backing for anonymous communication. Anonymous communication stays away from the outcome of personality uncovering issues. The different application areas of mysterious correspondence are: understanding restorative records (patient medical report), long range interpersonal communication (social networking), electronic voting, email [1]. The exponential headway in the field of data innovation and electronic correspondence offered climbs to new applications in the range of voting. There is an in number slant towards moving to Internet Voting - at any rate among the lawmakers - keeping in mind the end goal to upgrade voter comfort, expand voter certainty and voter turnout. Be that as it may, there are not kidding mechanical and social viewpoints that make Internet Voting infeasible in the unmistakable future. Along these lines, numerous technologists have recommended that remote survey site electronic voting, where the voter can vote at any survey site (not just his home district survey site), is by all accounts the best venture forward as it gives better voter accommodation, however in the meantime, does not bargain security. A study on the best in class in Electronic Voting, including the different works done in Internet Voting (and the contentions against its utilization), and also in electronic survey website voting were completed to look at the preferences and disservices of the framework

Electronic voting alludes to the utilization of PCs or modernized voting gear to cast tickets in a decision. Now and again, this term is utilized all the more particularly to allude to voting that happens over the Internet. PC frameworks can be utilized to enlist voters, count tickets, and record votes [1]. We can execute the e voting framework utilizing the electronic voting framework casing cooperates with the idea of anonymous communication. To separate anonymous ID assignment from anonymous communication, consider a circumstance where gatherings wish to show their information by and large, however secretly, in openings on an outsider site. The IDs can be utilized to appoint the spaces to clients, while anonymous communication can permit the gatherings to cover their characters from the outsider. In an alternate application, it is conceivable to utilize secure aggregate to permit one to quit of a reckoning heretofore on the premise of specific tenets in measurable revelation impediment or amid a processing and even to do as such in a mysterious way. Then again, next to no is known regarding routines permitting orgs to withdraw of a safe processing in light of the consequences of the examination, if they feel that those outcomes are excessively enlightening about their information [2].

\section{LITERATURE SURVEY}

\subsection{Anonymous Communication}

The anonymous communication investigates the association between imparting mysteries in a mysterious way, circulated secure multiparty calculation and mysterious ID task. The utilization of the expression "unknown" here contrasts from its significance in examination managing symmetry softening and pioneer decision up anonymous systems. Our system is not unknown and the members are identifiable in that they are known to and can be tended to by the others. Methods for assigning and using sets of pseudonyms have been developed for anonymous communication in mobile networks. The systems grew in these works for the most part oblige a trusted head, as composed, and their finished items by and large contrast from our own in structure and/or in measurable properties. The calculations AIDA appropriates a processing among the hubs creating a change of picked with a uniform likelihood of from the arrangement of all changes of where will know just [2]. Stage can be created by the calculation intended for metal porker. Metal porker calculations are more perplexing in nature and it utilize cryptographic strategy. Here, the participants are semi-honest, also known as passive or honest-but-curious, and execute their required protocols faithfully.

Mysterious correspondence is conceivable by offering straightforward number information on top of secure total. The offering calculation will be utilized at every emphasis of the calculation for unknown ID task [1]. The AIDA calculation and secure whole can oblige a variable and unbounded number of emphases. Expanding a parameter in the calculation will decrease the quantity of expected rounds. In any case, the primary calculation of mysterious correspondence obliges explaining a polynomial with coefficients taken from a limited field of numbers modulo a prime. That undertaking limit the level to which can be essentially raised.

Secure aggregate calculation is utilized to register and impart just the normal of information things in the information base, 
for example, number of healing center gained contaminations, without uncovering the estimation of this information thing for any individual from the gathering who taking part in the multiparty reckoning. In the event that we have a safe correspondence channel is accessible, it ought to be basic and asset escalated then secure whole can be executed.

Consider a circumstance where a gathering of hubs wishes to impart genuine estimations of information things from their database as opposed to depending on just measurable subtle elements then power total calculation together with secure entirety is more suitable. Force entirety is a symmetric capacity. Unknown information offering to power aggregates is carried out in way that the force wholes can be gathered and imparted utilizing a solitary round of secure entirety. Here the information is transmitted as an exhibit. It utilizes vectors for transmitting and accepting information.

\subsection{Voting Over Internet}

The Caltech/MIT Voting Technology Project started to exist keeping in mind the end goal to create another voting innovation to keep a repeat of the issues that happened in the 2000 U. S. Presidential Elections[7]. The fundamental point of that work was find the greatness of the issues, their underlying drivers and how innovation can lessen them. They address an extensive variety of "What is" issues including voting strategies, voting gear, voter enrollment, surveying spots, non-attendant and early voting, poll security, expense and open account of decisions, and so on. They then propose a novel "What could be" structure for voting innovation (that moves far from solid voting structures), and suggest that a methodology for development be setup. The system is "A Modular Voting Architecture ("Frogs")" in which vote era is performed independently from vote throwing, and the "Frog" structures a lasting review trail, the significance of which can't be over-pushed. Here, the vote era machine can be restrictive while the vote throwing machine must be open-source and altogether confirmed and ensured for rightness and security. At long last, the report gives an arrangement of transient and long haul suggestions on the different issues identified with voting.

In "Electronic Voting", Rivest addresses a few issues like the "safe stage issue" and the likelihood of giving a receipt to the voter. He additionally gives some individual suppositions on a large group of issues including the striking divergence between e-trade and e-voting, the threats of foes performing computerized, wide-scale assaults while voting from home, the requirement for great straightforwardness of voting supplies, the significance of review trails, support for debilitated voters, security issues of non-attendant tickets, and so on.

The NSF Internet Voting Report addresses the attainability of distinctive manifestations of Internet voting from both the specialized and sociology viewpoints, and characterizes an examination plan to seek after if Internet voting is to be feasible later on. It gathers Internet voting frameworks into three general classifications as follows:

- Poll-site Internet voting: It offers the guarantee of more noteworthy comfort and proficiency in that voters could cast their tallies from any survey website, and the counting procedure would be both quick and certain. All the more significantly, since decision authorities would control both the voting stage and the physical environment, dealing with the security dangers of such frameworks is doable
- Kiosk voting: Voting machines would be found far from conventional surveying spots, in such helpful areas as shopping centers, libraries, or schools. The voting stages would in any case be under the control of race authorities, and the physical environment could be altered as required and checked (e.g., by decision authorities, volunteers, or even cams) to address security and protection concerns, and anticipate compulsion or different manifestations of mediation.

- Remote Internet voting: It tries to amplify the comfort and access of the voters by empowering them to cast polls from essentially any area that is Internet available. While this idea is alluring and offers noteworthy profits, it additionally postures generous security dangers and different concerns with respect to urban society. Present and close term advancements are deficient to address these dangers.

The venture exhibits a few discoveries on the practicality of each of these classes and gives research suggestions to the long haul future. It then recognizes criteria for decision frameworks. At last, it addresses the innovative issues (counting voting framework vulnerabilities, unwavering quality, testing, accreditation and principles, particulars of source code, stage similarity, and mystery, and so forth.) and sociology issues, (for example, voter investment, voter get to, the race process, voter data, deliberative and agent majority rule government, group and character of decisions, dissemination of parts, legitimate concerns, voter enlistment, and so on.)

The California Internet Voting Report proposes a technique of transformative instead of progressive change towards accomplishing the objective of giving voters the chance to cast their tickets whenever from wherever through the Internet. It characterizes four unique Internet voting models Internet voting at voter's surveying spot, Internet voting at any surveying spot, Remote Internet voting from County PCs or stands, Remote Internet voting from any Internet association and the comparing specialized and configuration prerequisites that must be met when actualizing any of the stages. It addresses the preferences, execution and security issues of each of the four stages. They accept that extra specialized developments are vital before remote Internet voting can be broadly executed as a valuable device to enhance investment in the decisions procedure and that current innovation however would take into account the execution of new voting frameworks that would permit voters to cast a tally over the Internet from a PC at any of various region controlled surveying places in a province. The discoveries and proposals of the team on arrangement issues are additionally presented

A far reaching overview of e-voting innovation has been given in "e-Voting Security Study" [11]. It gives an overview of late scholarly and business extends in the range, notwithstanding the region's conspicuous scholastics' close to home perspectives and affirmations in regards to the issues. It distinguishes dangers, potential wellsprings of assault and conceivable routines for assault in such voting frameworks. It additionally distinguishes security destinations and prerequisites of an electronic voting framework.

The establishment of a great part of the scholarly work in the territory of remote voting is a paper by Fujioka, Okamoto and Ohta (FOO). It gives a scientific system for a protected race that includes an executive, and a counter and the voter associated by an unknown channel. Essentially centered activities expand on the visually impaired voting convention proposed in this paper. Sensus [13] uses blind marks to 
guarantee that just enlisted voters can vote and that every enrolled voter votes precisely once, while in the meantime keeping up voter's security. It permits voters to confirm autonomously that their votes were checked effectively and secretly challenge the outcomes, I cases the votes are miscalculated. An alternate task called E-VOX at MIT actualizes a disentangled, easy to use adaptation of the FOO system utilizing Java, Netscape and JDBC (Java Database Connectivity). This framework is still included in showing and examines and was utilized for an Undergraduates Association race at MIT in 1999. "Various Administrators for Electronic Voting" enhances this further by disseminating the power among different chairmen to avert vote manufacturing.

"An untraceable, generally evident voting plan" exhibits a remote voting plan that applies the strategy of blinded signature to a voter's vote so it is unthinkable for anybody to follow the tally back to the voter. They attain to the sought properties of protection, widespread obviousness, comfort and untraceability, yet to the detriment of receipt-freeness.

The Electronic Polling System for Remote Voting Operations undertaking explores broadband versatile interchanges taking into account the UMTS standard for giving the E-Poll system with the obliged data transfer capacity and security. This makes it conceivable to utilize E-Poll stands anyplace, inside a private, dependable and secured system. The voterdistinguishment framework is taking into account an imaginative keen card with an inserted biometric finger impression peruser, which performs voter distinguishment with total security. An ergonomic booth encourages use by impaired individuals. The FREE e-majority rules system venture is committed to making the GNU.FREE Internet Voting framework furthermore bolstering Free Software, which is non-divided and non-business in source. It shows a framework for secure electronic voting which does not depend on industrious system associations between surveying spots and the vote-counting server. They fabricate the framework on a separated (or, all the more precisely, an irregularly joined) environment, which acts well without system network[7,11,14].

"Security Criteria for Electronic Voting" thinks of some as essential criteria for privacy, uprightness, accessibility, dependability, and confirmation for PC frameworks included in electronic voting. After an evaluation of the feasibility of those criteria, it presumes that, operationally, a large portion of the criteria are characteristically unsatisfiable with any significant confirmation. Rubin distinguishes the new dangers realized by presenting the best in class innovation into the race process, which may not be worth taking. The significant security dangers recognized incorporate those at the voting stage - including vindictive payload (assault programs, remote organization and observing toolboxs, and so forth.) and conveyance component (worms, infections and bugs, dynamic substance downloaded naturally, and so on.) - and the correspondences foundation - including (circulated) dissent of administration assault, DNS server assault, and so on the framework recognizes security issues in social building and in utilizing particular devices

Computer scientists who have done work in, or are interested in, electronic voting all seem to agree on two things: Internet voting does not meet the requirements for public elections ,Currently widely-deployed voting systems need improvement
Voting on the Internet utilizing daily PC's offers just feeble security, yet its principle disservices are in the territories of secrecy and insurance against intimidation and/or vote offering. It's such a really awful thought, to the point that there is by all accounts no believable scholastic push to send it whatsoever. The Presidential decisions of 2000 brought national consideration regarding issues with current American routines for throwing and including votes open races. The vast majority accept that the current framework ought to be changed; there is much contradiction on how such changes ought to be made.

Neumann [9] gives a rundown of recommendations for "bland voting criteria" which proposes that a voting framework ought to be so difficult to mess with thus impervious to disappointment that no business framework is liable to ever meet the necessities, and adding to a suitable custom framework would be to a great degree troublesome and restrictively lavish. Rebecca Mercuri $[9,14]$ created the Mercuri system for electronic voting. A basic part of this system is very much alike to the Caltech/MIT proposition: a voting machine must create comprehensible hardcopy paper results, which can be confirmed by the voter before the vote is thrown, and physically related later if vital. Her rationality and Neumann's are fundamentally the same; truth be told, they've composed papers together on the subject. David Chaum presents an extremely fascinating plan [10], whereby voters could get receipts for their votes. This receipt would permit them to know whether their votes were incorporated in the last count or not, and to demonstrate that they voted without uncovering any data about how they voted. The security of this plan relies on upon visual cryptography grew by Naor and Shamir, and on voters haphazardly picking one of two bits of paper. Mercuri and Neumann[10] advocate the utilization of this strategy in electronic voting frameworks. Dr. Michael Shamos of CMU gives a sharp counterpoint [11] to Neumann and Mercuri's perspectives. Shamos is likewise a great deal less inspired with paper tallies than are Neumann and Mercuri. He puts a lot of confidence in decentralization to make misrepresentation hard to submit and simple to distinguish. Dr. Shamos even likes DRE machines.

\section{CONCLUSION}

Electronic voting framework which is more secured than the manual voting, to dodge future reoccurrence of difference amongst the individuals. Over whelming proof globally, has demonstrated that electronic voting framework can promise a valid and solid decision. This tries to expand the proficiency of voting methodology. Grow more secure databases and systems before setting out on e-voting; there ought to be appropriation of circulated encryption procedures with the end goal of secured information transmission; there ought to be utilization of biometric catching gadgets, which will serve as a method for voter's verification; there ought to be satisfactory and fitting open illumination before the framework is completely executed.

\section{ACKNOWLEDGMENT}

I hereby express my deep and sincere gratitude to my guide, Mrs. Anitha Sandeep for all the help and guidance offered to me to make this study fruitful one. I extend my sincere thanks to Prof. Raju K Gopal for all the support rendered to me make this study.

\section{REFERENCES}

[1] Larry A. Dunning, and Ray Kresman, "Privacy Preserving Data Sharing With Anonymous ID 
Assignment" IEEE Transactions On Forensics And Security ,vol:8, no:2,pp 402-413 year 2013

[2] Caltech/MIT Voting Technology Project (VTP) Report "Voting - What is, What Could be,", July 2001

[3] J.W. Yoon and H. Kim, "A perfect collision-free pseudonym system," IEEE Commun. Lett., vol. 15, no. 6, pp. 686-688, Jun. 2011.

[4] A. Friedman, R. Wolff, and A. Schuster, "Providing kanonymity in data mining," VLDB Journal, vol. 17, no. 4, pp. 789-804, Jul. 2008.

[5] C. Clifton, M. Kantarcioglu, J. Vaidya, X. Lin, and M. Y. Zhu, "Tools for privacy preserving distributed data mining," ACM SIGKDD Explorations Newsletter, vol. 4, no. 2, pp. 28-34, Dec. 2002.

[6] R. Canetti, "Security and composition of multi-party cryptographic protocols," J. Cryptol., vol. 13, no. 1, pp. 143-202, 2000

[7] The Caltech-MIT Voting Technology Project "A Preliminary Assessment of the Reliability of Existing Voting Equipment ", March 30, 2001 (revised).
Available

at http://www.vote.caltech.edu/Reports/index.html)

[8] Lorrie Cranor's Voting Papers,. Lorrie Faith Cranor. http://lorrie.cranor.org/pubs/voting.html

[9] "A Better Ballot Box?" Rebecca Mercuri, IEEE Spectrum, Volume 39, Number 10, October 2002.

[10] Security Criteria for Electronic Voting,. Peter Neumann, presented at the 16th National Computer Security Conference Baltimore, Maryland, September 20-23, $1993 . \quad$ Available http://www.csl.sri.com/users/neumann/ncs93.html

[11] Secret-Ballot Receipts and Transparent Integrity,. David Chaum, draft. Available at http://www.vreceipt.com/article.pdf

[12] Electronic Voting - Evaluating the Threat,. Michael Ian Shamos, CFP '93.

[13] Available http://www.cpsr.org/conferences/cfp93/shamos.html

[14] Electronic Voting,. $\quad$ Rebecca Mercuri. http://www.notablesoftware.com/evote.html 